\title{
Suboptimal Weight Loss and Weight Regain after Gastric Bypass Surgery-Postoperative Status of Energy Intake, Eating Behavior, Physical Activity, and Psychometrics
}

\author{
Tina Amundsen ${ }^{1}$ - Magnus Strømmen ${ }^{1,2,3}$ - Catia Martins ${ }^{1,2}$
}

Published online: 2 December 2016

(C) The Author(s) 2016. This article is published with open access at Springerlink.com

\begin{abstract}
Background Suboptimal weight loss (SWL) and weight regain (WR) after gastric bypass surgery (GB) remains poorly understood.

Objectives This study aims to compare GB patients experiencing SWL or significant WR (SigWR) with successful controls, regarding postoperative food intake, eating behavior, physical activity (PA), and psychometrics.

Methods Forty-nine patients with $>1$ year post-surgery were classified as either experiencing SWL (excess body weight loss, EWL, $<50 \%, n=22$ ) or SigWR (total weight regain $\geq 15 \%, n=38$ ), with respective control groups. Energy intake (EI) was measured with a Food Frequency Questionnaire, eating behavior using the Dutch Eating Behavior Questionnaire and the Three-Factor Eating Questionnaire, and PA using both SenseWear Armbands and the International Physical Activity Questionnaire. Eating disorders, depression, and quality of life (QoL) were measured using the Eating Disorder Examination Questionnaire, Beck Depression Inventory II, and Impact of Weight on Quality of Life, respectively.

Results EI, macronutrient distribution, and meal frequency were similar among groups. However, disinhibited eating
\end{abstract}

Catia Martins

catia.martins@ntnu.no

1 Centre for Obesity Research (ObeCe), Department of Surgery, St. Olavs Hospital - Trondheim University Hospital, Prinsesse Kristinas gate 3, 7030 Trondheim, Norway

2 Obesity Research Group, Department of Cancer Research and Molecular Medicine, Faculty of Medicine, Norwegian University of Science and Technology, Trondheim, Norway

3 Department of Neuroscience, Faculty of Medicine, Norwegian University of Science and Technology, Trondheim, Norway behavior score was higher, while most subcategories from IWQOL were significantly lower in both SWL and SigWR groups compared with their respective controls. PA was significantly lower in the SWL and SigWR groups compared with the respective controls. There were no differences between groups regarding depression.

Conclusions Lower PA levels, disordered eating behavior and lower QoL are associated with unsuccessful weigh loss outcome after GB surgery. Longitudinal studies are needed to clarify the potential causal relationship between the previously described variables and SWL/SigWR after GB.

Keywords Morbid obesity · Gastric bypass · Suboptimal weight loss $\cdot$ Weight regain $\cdot$ Energy intake $\cdot$ Eating behavior . Physical activity

\section{Introduction}

Gastric bypass (GB) is a common bariatric procedure for patients with severe obesity (BMI $\geq 40 \mathrm{~kg} / \mathrm{m}^{2}$ or BMI $\geq 35 \mathrm{~kg} / \mathrm{m}^{2}$ with comorbidities) [1]. The long-term effects of GB are well documented, both in terms of weight loss, improvement or resolution of obesity-related comorbidities, and risk factors [2], as well as improvement in quality of life (QoL) [3]. The exact mechanisms mediating the success of GB are still unknown, but changes in appetite hormones, bile acids, and microbiota have all been suggested [4].

Unfortunately, a subset of patients experience suboptimal weight loss (SWL), defined as either $\leq 50$ or $\leq 40 \%$ excess body weight loss (EBWL) after GB surgery [5-8]. The prevalence of SWL increases over time. Approximately $20 \%$ of patients experience SWL 1 year after surgery [7, 8], while this proportion can reach as much as $33 \%$ at 10 years of follow-up [9]. In addition, weight regain (WR) has been reported in approximately $50 \%$ of 
GB patients within 2 years after surgery [10], with $15 \%$ gaining $\geq 15 \%$ weight from nadir [11].

Few studies have tried to identify factors responsible for SWL or WR after GB [5, 6, 12, 13]. Preoperative (greater initial BMI, male gender, and diabetes mellitus type 2 [6]) and postoperative factors (low dietary adherence [12] and high soda consumption [13]) have previously been associated with SWL or WR after GB. Overconsumption of energy-dense food postoperatively has also been associated with WR [14]. Eating behavior (including restrained, disinhibited, external, and emotional eating, and hunger perception) have shown to improve after GB [15, 16], and some studies suggest that preoperative scores may predict weight loss outcomes $[12,17]$. Additionally, health-related QoL improves in most patients after bariatric surgery, but poor weight loss outcome, along with postoperative depression, can influence QoL negatively $[18,19]$.

More research is, therefore, required to improve our understanding of SWL and WR after GB, so that preventive strategies can be developed.

The primary aim of this study was to compare GB patients experiencing SWL or significant WR (SigWR) with successful controls, regarding postoperative food intake, eating behavior, and physical activity (PA). The secondary aim was to determine if an association exists between SWL/WR and QoL, depressive symptoms, and disordered eating.

\section{Subjects and Methods}

\section{Study Design}

This was a case-control study in which adult patients with SWL or significant WR (SigWR) after GB were compared with respective controls achieving an acceptable weight loss (AWL) and non-significant weight regain (NWR).

\section{Subjects}

Participants were GB patients, who had undergone surgery a minimum of 1 year prior to this study. Invitation letters were sent to patients who complied to follow-up meetings at the Obesity Clinic at St. Olavs Hospital (Trondheim, Norway).

Exclusion criteria included pregnancy, enrollment in another obesity treatment, previous bariatric surgery other than GB, previous revisional bariatric surgery, known past or ongoing substance abuse, history of severe psychological disorder, severe eating disorder, and current medication known to affect body weight.

This study was conducted according to the guidelines laid down in the Declaration of Helsinki and was approved by the Ethics Committee (2012/1884, Central-Norway). Written informed consent was obtained from all participants before enrollment.

\section{Detailed Protocol}

Participants were invited to St. Olavs Hospital for a screening interview, including anthropometric measurements and various questionnaires (all self-administered). Measurement of PA levels was, in addition to a questionnaire, also assessed with armbands. Participants were classified as either experiencing SWL (excess body weight loss $(\mathrm{EBWL})<50 \%, n=21$ ) or AWL ( $n=27$ ) (control group), and SigWR (total weight regain $($ TWR $)>15 \% n=38)$ or NWR $($ TWR $\leq 15 \%, n=11)$ (control group). A detailed description of how \%EBWL and \% TWR were calculated can be found below. The four groups were overlapping, meaning that participants could serve as a case in one group and a control in another group.

\section{Variables Collected}

\section{Anthropometrics}

Height was measured without shoes, using a stadiometer (Seca, GmbH \& Co., Germany) to the nearest $0.5 \mathrm{~cm}$. Weight was measured to the nearest $0.1 \mathrm{~kg}$, with light clothing and without shoes, using a digital scale (Seca, $\mathrm{GmbH} \& \mathrm{Co}$, Germany).

Information on preoperative weight (operation day) was gathered from the participants' hospital records. Lowest achieved weight after surgery was self-reported, usually recorded at a follow-up meeting 6 months to 2 years postoperatively.

The percentage of EBWL was calculated by dividing weight loss $(\mathrm{kg})$ by excess weight [(preoperative weight - ideal weight $) \times 100$ ]. Ideal weight was defined as a weight corresponding to a BMI of $25 \mathrm{~kg} / \mathrm{m}^{2}$. Total weight regain (TWR) in percentage was calculated as (100/(preoperative weight - nadir weight) $\times$ weight regain $(\mathrm{kg})$. Total WL was calculated as preoperative weight - current weight, while actual WL as preoperative weight - nadir weight.

\section{Energy Intake and Eating Behavior}

A 23-item Food Frequency Questionnaire (FFQ), validated on the Norwegian population [20], was used to collect data on total energy intake (EI), energy percentage $(\% \mathrm{E})$ provided by each macronutrient, intake of various food groups, and meal frequency. Participants were asked to have the last year in mind when reporting food intake. The FFQs were analyzed by the Medical Faculty at the University of Oslo, Norway.

Two questionnaires were used to measure eating behavior: the Three-Factor Eating Questionnaire (TFEQ) and the Dutch Eating Behavior Questionnaire (DEBQ). The TFEQ measures three different dimensions: restraint, disinhibition, and hunger [21]. The DEBQ also has three dimensions: emotional, external, and restrained eating [22]. 


\section{Physical Activity}

PA levels were measured with armbands (SenseWear Armband; BodyMedia, Pittsburgh, USA) and estimated by the International Physical Activity Questionnaire (IPAQ, Norwegian version) [23]. Participants were asked to use the armbands for 7 days. To be included in the analyses, each participant needed a minimum of 6 days of armband data, including two weekend days. One valid day was equal to $1368 \mathrm{~min}$ of data $(95 \%$ of a 24 -h period). The following variables were analyzed: average metabolic equivalent (MET); number of steps; time spent on sedentary, light, moderate, and vigorous activity; and total physical activity duration (min/day). Sedentary time was assumed as <1.5 MET, light as 1.5-2.9 METs, and moderate and vigorous activity as a MET between 3.0-5.9 and 6.0-8.7, respectively [24-27].

\section{Psychometrics}

Eating disorders were assessed by the Eating Disorder Examination (EDE)-Q 6.0 (Norwegian version) questionnaire [28]. EDE-Q has four subscales: dietary restraint, eating concern, weight concern, and shape concern, including a global score which is the average of the subscales. A global score of 4.0 has been suggested, but not validated, as a cut-off for disordered eating behavior [29].

Depression was assessed with the Beck Depression Inventory II (BDI-II, Norwegian version) [30]. The score ranges from 0 to 63 , and the severity of depression is categorized in four groups (0-13 minimal, 14-19 mild, 20-28 moderate, and 29-63 severe depression).

QoL was assessed using Impact of Weight on Quality of Life-Lite (IWQOL-Lite, Norwegian version) [31].

\section{Statistical Analysis}

Statistical analysis was carried out using SPSS 22.0 (SPSS Inc., Chicago, IL, USA). Data is reported as mean \pm standard deviation (SD) for normally distributed variables and as median and interquartile range (IQR) for non-normally distributed variables. Statistical significance was assumed at $p<0.05$. Differences between groups were assessed by an independent samples $t$ test for normally distributed continuous variables and Mann-Whitney $U$ test for the non-normally distributed continuous variables (variables from FFQ, IWQOL-Lite, armbands, and IPAQ). Chisquare, along with Fisher's exact test, was used for categorical variables.

The Benjamini-Hochberg method, which controls for the false discovery rate [32], was used to adjust for multiple comparisons. $P$ values for group comparisons are shown both as unadjusted and adjusted.

\section{Results}

\section{Subject Characteristics}

A total of 51 participants met for the initial interview. Of those, two participants withdrew for unknown reasons, giving a total of 49 participants. The subject characteristics are displayed in Table 1.

The total sample consisted mainly of women (82\%), with an average age of 46 years, preoperative BMI of $44.1 \mathrm{~kg} / \mathrm{m}^{2}$, and postoperative follow-up time of 5 years. There were no significant differences in preoperative data or postoperative follow-up between any of the groups. TWR\% was significantly higher in the SWL and SigWR groups, while total WL and \%EBWL were significantly lower in the SWL and SigWR groups. No

Table 1. Characteristics of each group

\begin{tabular}{|c|c|c|c|c|c|c|}
\hline Characteristics & $\operatorname{SigWR}(n=36)$ & $\operatorname{NWR}(n=11)$ & $p$ value & SWL $(n=22)$ & $\operatorname{AWL}(n=27)$ & $p$ value \\
\hline Female sex $n(\%)$ & $31(81.6)$ & $11(100)$ & $0.124(0.232)$ & $18(81.8)$ & $24(88.9)$ & $0.482(0.657)$ \\
\hline Age (years) & $45.5 \pm 7.2$ & $51.5 \pm 7.5$ & $0.02(0.064)$ & $46.5 \pm 6.9$ & $47.1 \pm 8.2$ & $0.815(0.937)$ \\
\hline Postoperative follow-up (months) & $59.6 \pm 26.0$ & $53.9 \pm 20.4$ & $0.503(0.663)$ & $63.4 \pm 27.6$ & $54.2 \pm 21.9$ & $0.2(0.358)$ \\
\hline Preoperative BMI $\left(\mathrm{kg} / \mathrm{m}^{2}\right)$ & $45.1 \pm 6.1$ & $44.7 \pm 7.6$ & $0.877(0.937)$ & $46.2 \pm 6.6$ & $44.0 \pm 6.3$ & $0.227(0.385)$ \\
\hline Actual WL (pre-nadir weight) $\%$ & $56.6 \pm 17.2$ & $53.9 \pm 17.8$ & $0.233(0.385)$ & $59.1 \pm 17.6$ & $53.5 \pm 16.7$ & $0.006(0.027)$ \\
\hline Total WL (pre-current weight) \% & $18.8 \pm 10.0$ & $36.3 \pm 6.4$ & $<0.001(0.002)$ & $14.7 \pm 8.7$ & $28.8 \pm 10.1$ & $<0.001(<0.001)$ \\
\hline TWR \% & $43.7 \pm 30.6$ & $6.8 \pm 6.0$ & $<0.001(<0.001)$ & $56.3 \pm 34.3$ & $18.4 \pm 13.0$ & $<0.001(<0.001)$ \\
\hline EBWL \% & $44.8 \pm 22.7$ & $86.1 \pm 22.5$ & $<0.001(<0.001)$ & $29.8 \pm 15.6$ & $73.8 \pm 19.7$ & $<0.001(<0.001)$ \\
\hline
\end{tabular}

Data presented as mean $\pm \mathrm{SD} . P$ values are shown as unadjusted (adjusted for multiple comparisons)

SigWR significant weight regain, $N W R$ non-significant weight regain, $S W L$ suboptimal weight loss, $A W L$ acceptable weight loss, $W L$ weight loss, $T W R$ total weight regain, $E B W L$ excess body weight loss 
significant difference between any of the groups was found regarding actual WL (Table 1).

\section{Food Intake}

Table 2 displays EI and \% E from each macronutrient derived from the FFQ. No significant differences between none of the four groups were observed for EI or \% $\mathrm{E}$ coming from carbohydrates, protein, and fat. The number of meals per day, breakfast frequency, snacking frequency, and intake of different food groups (data not shown) were also similar across all groups.

\section{Eating Behavior}

Scores derived from the TFEQ and DEBQ are outlined in Table 3. There was a tendency for the disinhibition score from the TFEQ to be higher in SWL and SigWR groups compared to their respective controls. No other dimensions reached statistical significance.

\section{Physical Activity}

Table 4 displays self-reported (IPAQ) and objectively measured (SenseWear Armbands) PA levels. Time spent on walking and total physical activity duration (IPAQ) were significantly lower in the SWL group. Regarding data derived from armbands, daily average MET, time spent on light PA, and total PA duration were significantly lower in the SWL and SigWR groups.

\section{Psychometrics}

Table 5 outlines psychometrics derived from the IWQOLLite, EDE-Q, and BDI-II. Median score for most subcategories in IWQOL-Lite were significantly lower in SWL and SigWR groups compared with respective controls.
Regarding eating disorders, the only significant difference found was for weight and shape concern, which were higher in the SWL group. No significant difference between groups was found on the BDI-II total score (or the percentage of participants in each depression group).

\section{Discussion}

In the present study, participants experiencing either SWL or SigWR were less physically active and presented with higher disinhibition and weight and shape concern scores, compared to participants with AWL or NWR. Moreover, they also presented with an overall lower QoL. In accordance with our expectations, several self-reported and objectively measured PA variables were found to be significantly lower in the SWL and SigWR groups. This is in line with several other studies showing that PA is important in weight maintenance and prevention of weight regain after GB [33-35]. Participants who engage in $>150$ min of moderate to vigorous PA were previously found to have a greater \%EBWL than those who were less physically active 2-5 years after GB [36]. Self-reported moderate and total PA have also been reported to correlate positively with \%EBWL [37].

The American College of Sports Medicine (ACSM) recommends $>250$ min per week of moderate PA to prevent weight regain [38]. In the present study, the AWL group had an average of $431 \mathrm{~min}$ of moderate PA per week $(61.5 \mathrm{~min} /$ day), while the NWR group had $567 \mathrm{~min}$ per week $(81.0 \mathrm{~min} /$ day), which is well above the ACSM's recommendation. Time spent on moderate activity in the SWL and SigWR groups was almost half of that seen in the respective control groups, but in accordance with the recommendations (on average $266 \mathrm{~min}$ / week). These results suggest that the present PA recommendations proposed by the ACSM to prevent weight regain may not be sufficient after GB.

Contrary to our expectations, no significant differences in total EI, macronutrient distribution, intake of different food

Table 2. Energy intake and \% $\mathrm{E}$ from macronutrients (FFQ)

\begin{tabular}{|c|c|c|c|c|c|c|}
\hline$\% \mathrm{E}$ macronutrients & SigWR $(n=36)$ & $\operatorname{NWR}(n=11)$ & $\begin{array}{l}p \\
\text { value }\end{array}$ & SWL $(n=21)$ & AWL $(n=26)$ & $\begin{array}{l}p \\
\text { value }\end{array}$ \\
\hline Energy intake (kcal) & $1910.0(620.2)$ & $1670.0(582.0)$ & $0.364(0.523)$ & $1879.0(1129.0)$ & $1849.0(1104.0)$ & $0.915(0.937)$ \\
\hline$\% \mathrm{E}$ of protein & $17.1(5.9)$ & $16.8(4.7)$ & $0.268(0.427)$ & $16.7(2.3)$ & $17.4(4.4)$ & $0.571(0.722)$ \\
\hline$\% \mathrm{E}$ of carbohydrates & $41.8(8.8)$ & $43.4(11.8)$ & $0.853(0.937)$ & $42.2(6.5)$ & $41.1(9.1)$ & $0.716(0.867)$ \\
\hline$\% \mathrm{E}$ of added sugar & $6.0(6.3)$ & $4.9(2.8)$ & $0.911(0.937)$ & $7.1(6.0)$ & $5.1(5.5)$ & $0.831(0.937)$ \\
\hline$\% \mathrm{E}$ of fat & $36.6(10.3)$ & $36.5(7.8)$ & $0.611(0.750)$ & $37.1(10.6)$ & $36.3(8.7)$ & $0.585(0.729)$ \\
\hline$\%$ E of fiber & $2.3(1.0)$ & $2.5(0.8)$ & $0.931(0.942)$ & $2.3(0.8)$ & $2.4(1.0)$ & $0.915(0.937)$ \\
\hline$\% \mathrm{E}$ of alcohol & $0.3(2.0)$ & $2.0(7.8)$ & $0.057(0.137)$ & $0.0(0.6)$ & $0.9(3.0)$ & $0.015(0.052)$ \\
\hline
\end{tabular}

Data presented as median (interquartile range). $P$ values are shown as unadjusted (adjusted for multiple comparisons)

SigWR significant weight regain, $N W R$ non-significant weight regain, $S W L$ suboptimal weight loss, $A W L$ acceptable weight loss, $\% E$ energy percentage 
Table 3. Eating behavior (TFEQ and DEBQ)

\begin{tabular}{|c|c|c|c|c|c|c|}
\hline $\begin{array}{l}\text { Eating } \\
\text { behavior }\end{array}$ & $\begin{array}{l}\text { SigWR } \\
(n=38)\end{array}$ & $\begin{array}{l}\text { NWR } \\
(n=11)\end{array}$ & $\begin{array}{l}p \\
\text { value }\end{array}$ & $\begin{array}{l}\text { SWL } \\
(n=22)\end{array}$ & $\begin{array}{l}\text { AWL } \\
(n=27)\end{array}$ & $\begin{array}{l}p \\
\text { value }\end{array}$ \\
\hline \multicolumn{7}{|l|}{ TFEQ } \\
\hline Restraint & $10.8 \pm 3.9$ & $10.5 \pm 5.1$ & $\begin{array}{l}0.853 \\
(0.937)\end{array}$ & $9.9 \pm 3.9$ & $11.4 \pm 4.3$ & $0.233(0.385)$ \\
\hline Disinhibition & $7.9 \pm 3.3$ & $4.5 \pm 4.0$ & $\begin{array}{l}0.02 \\
(0.064)\end{array}$ & $8.1 \pm 2.9$ & $5.6 \pm 3.7$ & $0.015(0.052)$ \\
\hline Hunger & $4.0 \pm 2.4$ & $3.9 \pm 2.7$ & $\begin{array}{l}0.844 \\
(0.937)\end{array}$ & $4.6 \pm 2.5$ & $3.5 \pm 2.3$ & $0.13(0.238)$ \\
\hline \multicolumn{7}{|l|}{ DEBQ } \\
\hline Emotional & $2.6 \pm 1.0$ & $2.3 \pm 0.8$ & $\begin{array}{l}0.392 \\
(0.562)\end{array}$ & $2.7 \pm 0.8$ & $2.5 \pm 1.1$ & $0.399(0.563)$ \\
\hline External & $2.8 \pm 5.7$ & $2.4 \pm 0.3$ & $\begin{array}{c}0.069 \\
0.145\end{array}$ & $2.9 \pm 0.5$ & $2.6 \pm 0.5$ & $0.062(0.137)$ \\
\hline Restraint & $2.9 \pm 0.5$ & $2.5 \pm 0.7$ & 0.050 .126 & $2.9 \pm 0.5$ & $2.7 \pm 0.6$ & $0.517(0.663)$ \\
\hline
\end{tabular}

Data presented as mean $\pm \mathrm{SD}$. $P$ values are shown as unadjusted (adjusted for multiple comparisons)

SigWR significant weight regain, $N W R$ non-significant weight regain, $S W L$ suboptimal weight loss, $A W L$ acceptable weight loss, TFEQ Three-Factor Eating Questionnaire, $D E B Q$ Dutch Eating Behavior Questionnaire groups, and meal/breakfast frequency were found between any of the groups. It needs to be emphasized, nevertheless, that the SigWR group had an energy intake which was $14.4 \%$ (240 kcal) higher than the NWR group and larger studies could potentially show significant differences between groups. Weight regain after GB has previously been associated with poor diet quality (higher caloric intake, sweets, snacks, and fatty foods) [33], while a lower daily EI has been found to correlate with \%EBWL $[39,40]$. However, two other studies reported no correlation between EI and \%EBWL after GB surgery [41, 42]. Given the larger postoperative BMI in the SWL and SigWR groups (assuming that participants were weight stable), a higher total EI would be expected in those groups. The fact that food intake was self-reported and may suffer from underreporting and the small sample size of the study may therefore have biased the results.

Disinhibition (TFEQ) score was found to be significantly higher in both the SWL and SigWR groups. Previous studies have shown inconclusive results and, unlike the present study, tend to present only preoperative scores $[12,17,43]$. Lower disinhibition and hunger scores (TFEQ) have been reported 1 year after adjustable gastric banding or vertical banded

Table 4. Physical activity levels from IPAQ and armbands

\begin{tabular}{|c|c|c|c|c|c|c|}
\hline Physical activity & $\operatorname{SigWR}(n=38)$ & $\operatorname{NWR}(n=11)$ & $\begin{array}{l}p \\
\text { value }\end{array}$ & $\operatorname{SWL}(n=22)$ & AWL $(n=27)$ & $\begin{array}{l}p \\
\text { value }\end{array}$ \\
\hline \multicolumn{7}{|l|}{ IPAQ (min/week) } \\
\hline Walking & $495.0(2054.2)$ & $792.0(3799.1)$ & $0.095(0.186)$ & $198.0(858.0)$ & $742.5(1930.5)$ & $0.005(0.024)$ \\
\hline Moderate activity & $0.0(480.0)$ & $0.0(840.0)$ & $0.885(0.937)$ & $0.0(240.0)$ & $120.0(480.0)$ & $0.321(0.493)$ \\
\hline Vigorous activity & $0.0(690.0)$ & $0.0(1680.0)$ & $0.383(0.558)$ & $0.0(60.0)$ & $0.0(960.0)$ & $0.209(0.3687)$ \\
\hline Total PA & $862.5(3048.3)$ & $2748.0(2560.5)$ & $0.043(0.112)$ & $334.5(1488.0)$ & $2232.0(3415.5)$ & $<0.001(0.005)$ \\
\hline SenseWear armbands & $\operatorname{SigWR}(n=25)$ & $\operatorname{NWR}(n=9)$ & & $\operatorname{SWL}(n=14)$ & $\operatorname{AWL}(n=20)$ & \\
\hline Daily average MET & $1.1(0.2)$ & $1.4(0.3)$ & $0.004(0.020)$ & $1.0(0.3)$ & $1.2(0.2)$ & $<0.001(0.005)$ \\
\hline Sedentary activity (min/day) & $1199.0(112.0)$ & $1118.0(205.5)$ & $0.03(0.086)$ & $1207.0(149.0)$ & $1136.0(140.0)$ & $0.061(0.137)$ \\
\hline Light activity (min/day) & $153.0(80.2)$ & $247.0(71.2)$ & $0.004(0.020)$ & $145.0(75.0)$ & $223.5(105.0)$ & $0.015(0.052)$ \\
\hline Moderate activity (min/day) & $41.0(39.5)$ & $81.0(100.0)$ & $0.06(0.137)$ & $35.0(46.0)$ & $61.5(49.0)$ & $0.056(0.137)$ \\
\hline Vigorous activity (min/day) & $0.0(1.2)$ & $0.0(5.0)$ & $0.489(0.657)$ & $0.0(1.0)$ & $0.0(4.0)$ & $0.743(0.875)$ \\
\hline Total PA duration (min/day) & $195.0(112.0)$ & $314.0(152.7)$ & $0.003(0.018)$ & $181.0(122.0)$ & $276.0(114.0)$ & $0.0007(0.031)$ \\
\hline Number of steps/day & $4919.0(3978.7)$ & $5686.0(5539.3)$ & $0.489(0.657)$ & $4777.5(3098.0)$ & $5688.0(3361.0)$ & $0.245(0.398)$ \\
\hline
\end{tabular}

Data presented as median (interquartile range). $P$ values are shown as unadjusted (adjusted for multiple comparisons)

SigWR significant weight regain, $N W R$ non-significant weight regain, $S W L$ suboptimal weight loss, $A W L$ acceptable weight loss, IPAQ International Physical Activity Questionnaire, MET total average metabolic equivalent of task. Sedentary activity: $<1.5$ MET (minutes). Light activity: 1.5-3.0 MET (minutes). Moderate activity: 3.1-5.9 MET (minutes). Vigorous activity: 6.0-8.7 MET (minutes). Physical activity duration: >1.5 MET (minutes) 
Table 5. Psychometrics (IWQOL, EDE-Q, and BDI-II)

\begin{tabular}{|c|c|c|c|c|c|c|}
\hline Questionnaire & $\operatorname{SigWR}(n=38)$ & $\operatorname{NWR}(n=11)$ & $\begin{array}{l}p \\
\text { value }\end{array}$ & $\operatorname{SWL}(n=21)$ & $\operatorname{AWL}(n=27)$ & $\begin{array}{l}p \\
\text { value }\end{array}$ \\
\hline \multicolumn{7}{|l|}{ IWQOL-Lite } \\
\hline Physical functioning & $82.9(26.7)$ & $95.4(7.3)$ & $0.004(0.020)$ & $79.5(30.6)$ & $88.6(13.6)$ & $0.002(0.014)$ \\
\hline Self-esteem & $66.0(56.2)$ & $96.4(14.0)$ & $0.014(0.052)$ & $58.9(41.0)$ & $85.7(25.0)$ & $<0.001(0.005)$ \\
\hline Sexual life & $87.5(51.5)$ & $100(0.0)$ & $0.065(0.140)$ & $75.0(46.8)$ & $100.0(18.7)$ & $0.013(0.05)$ \\
\hline Public distress & $85.0(35.0)$ & $100(0.0)$ & $0.03(0.086)$ & $82.5(37.5)$ & $100.0(15.0)$ & $<0.001(0.008)$ \\
\hline Work & $100(18.7)$ & $100(0.0)$ & $0.366(0.543)$ & $93.7(31.2)$ & $100.0(0.0)$ & $0.037(0.099)$ \\
\hline EDE-Q & & & & $\operatorname{SWL}(n=21)$ & $\operatorname{AWL}(n=26)$ & \\
\hline Restraint & $1.3 \pm 0.9$ & $1.1 \pm 1.3$ & $0.509(0.663)$ & $1.1 \pm 0.7$ & $1.4 \pm 1.2$ & $0.29(0.453)$ \\
\hline Eating concern & $0.9 \pm 1.0$ & $0.8 \pm 0.9$ & $0.732(0.874)$ & $0.9 \pm 1.0$ & $0.8 \pm 1.1$ & $0.883(0.937)$ \\
\hline Shape concern & $2.9 \pm 1.3$ & $2.0 \pm 1.8$ & $0.074(0.152)$ & $3.4 \pm 1.0$ & $2.2 \pm 1.6$ & $0.008(0.033)$ \\
\hline Weight concern & $2.6 \pm 0.9$ & $1.3 \pm 1.7$ & & $2.9 \pm 0.7$ & $1.8 \pm 1.4$ & $0.001(0.008)$ \\
\hline Global score & $1.9 \pm 0.8$ & $1.3 \pm 1.2$ & $0.074(0.152)$ & $2.0 \pm 0.6$ & $1.5 \pm 1.1$ & $0.111(0.212)$ \\
\hline BDI-II & $\operatorname{SWR}(n=38)$ & $\operatorname{NWR}(n=11)$ & & SWL $(n=22)$ & AWL $(n=27)$ & \\
\hline Total score & $9.0 \pm 7.9$ & $9.2 \pm 10.3$ & $0.946(0.946)$ & $11.9 \pm 8.6$ & $6.7 \pm 7.5$ & $0.029(0.086)$ \\
\hline Minimal depression, $n(\%)$ & $30(78.9)$ & $9(81.8)$ & & $15(68.2)$ & $24(88.9)$ & \\
\hline Mild depression, $n(\%)$ & $3(7.9)$ & $0(0.0)$ & & $2(9.1)$ & $1(3.7)$ & \\
\hline Moderate depression, $n(\%)$ & $4(9.1)$ & $1(10.5)$ & & $4(18.2)$ & $1(3.7)$ & \\
\hline Severe depression, $n(\%)$ & $1(2.6)$ & $1(9.1)$ & & $1(4.5)$ & $1(3.7)$ & \\
\hline
\end{tabular}

Data for EDE-Q and BDI-II total score presented as mean \pm SD. $P$ values are shown as unadjusted (adjusted for multiple comparisons). Depression categories presented as $n$ (\% within each group). Data for IWQOL presented as median (interquartile range)

SigWR significant weight regain, $N W R$ non-significant weight regain, $S W L$ suboptimal weight loss, $A W L$ acceptable weight loss

gastroplasty on those successful in weight loss (versus unsuccessful) [44]. To our knowledge, only one study has examined DEBQ and weight loss after GB [43]. Opposite to our results, the study found an inverse relationship between weight loss and emotional and external eating, but no relationship with restrained eating [43].

Regarding the EDE-Q, weight and shape concern were found to be significantly higher in both the SWL groups. However, all groups had average values below the cut-off for disordered eating. Still, the presence of a sub-clinical eating disorder cannot be fully excluded. Some studies have found that EDE-Q scores significantly improve after GB $[45,46]$. Very few studies have examined the relationship between disordered eating and unsuccessful weight loss after GB. Hrabosky and colleagues (2006) reported no correlation between the degree of weight loss and changes in EDE-Q scores postoperatively [45]. Another study also found improvements in different aspects of eating symptomatology (using EDI-3), but reported no correlation between such symptoms and weight loss after GB [47].

We found no significant differences in depressive symptoms between groups, and the average total score was in the minimal depression category for all. Several studies have reported an improvement in depressive symptoms after various bariatric surgery procedures, regardless of weight loss [42, 48-50]. However, Faulconbridge and colleagues (2013) reported a positive correlation between improvement in depression scores (BDI-II) postoperatively and the degree of weight loss [51]. Another study found preoperative BDI score to be positively correlated with the amount of weight lost 1 year after GB surgery [52].

Consistent with our expectations, most dimensions of the IWQOL-Lite were significantly lower in the SWL and SigWR groups, suggesting that those unsuccessful after GB have a lower QoL. A previous study has reported similar QoL improvements in both successful and unsuccessful weight loss participants ( $<50 \%$ EBWL) 8 years after GB [42]. A significant improvement in QoL was also reported in another study, using the SF-36 questionnaire (a generic QoL-assessment tool), with greater improvement seen in patients with the largest weight reduction [53].

It is important to note that our unsuccessful participants, regarding weight loss outcome after GB, regardless of the definition used (SWL based on EWL\% or SigWR), presented with no significant difference in actual WL (given as preoperative - nadir weight) compared with those successful (AWL and NWR). However, both the SWL and SigWR groups had a significantly lower total WL (given as preoperative - current weight) and a significantly higher TWR\%. Thus, successful weight loss outcome after GB surgery was due to the degree of weight regain and not actual weight loss. Limitations in this study include its small sample size, which may underpower it 
statistically, its cross-sectional design and subsequently the lack of preoperative measurements, and the fact that the lowest achieved weight was self-reported. Moreover, changes in post-surgical anatomy, which can affect weigh regain, were not looked at. Despite these limitations, the current findings are significant and can help clinicians better manage their patients.

In conclusion, lower PA levels, disordered eating behavior, and lower QoL are associated with unsuccessful weight loss outcome after GB, defined either as SWL or SigWR. Future studies, with a longitudinal design and larger sample sizes, are needed to clarify the causal relationship between SWL and WR after GB and the previously described variables.

Acknowledgements We acknowledge the funding support by the Centre for Obesity Research (ObeCe), Department of Surgery at St. Olavs Hospital (Trondheim, Norway), in partnership with the Norwegian University of Science and Technology (NTNU, Norway). Special thanks to Hege Tevik Bjøru for help with recruitment of participants and to Turid Follestad for help regarding statistical analysis.

\section{Compliance with Ethical Standards}

Conflict of Interest The authors declare that they have no competing interests.

Open Access This article is distributed under the terms of the Creative Commons Attribution 4.0 International License (http:// creativecommons.org/licenses/by/4.0/), which permits unrestricted use, distribution, and reproduction in any medium, provided you give appropriate credit to the original author(s) and the source, provide a link to the Creative Commons license, and indicate if changes were made.

\section{References}

1. Sjostrom L, Narbro K, Sjostrom CD, Karason K, Larsson B, Wedel $\mathrm{H}$, et al. Effects of bariatric surgery on mortality in Swedish obese subjects. N Engl J Med. 2007;357(8):741-52.

2. Sjostrom L, Lindroos AK, Peltonen M, Torgerson J, Bouchard C, Carlsson B, et al. Lifestyle, diabetes, and cardiovascular risk factors 10 years after bariatric surgery. $\mathrm{N}$ Engl J Med. 2004;351(26):2683-93.

3. Julia C, Ciangura C, Capuron L, Bouillot JL, Basdevant A, Poitou $\mathrm{C}$, et al. Quality of life after Roux-en-Y gastric bypass and changes in body mass index and obesity-related comorbidities. Diabetes Metab. 2013;39(2):148-54.

4. Sweeney TE, Morton JM. Metabolic surgery: action via hormonal milieu changes, changes in bile acids or gut microbiota? A summary of the literature. Best Pract Res Clin Gastroenterol. 2014;28(4): 727-40.

5. Campos GM, Rabl C, Mulligan K, Posselt A, Rogers SJ, Westphalen AC, et al. Factors associated with weight loss after gastric bypass. Arch Surg. 2008;143(9):877-83.

6. Melton GB, Steele KE, Schweitzer MA, Lidor AO, Magnuson TH. Suboptimal weight loss after gastric bypass surgery: correlation of demographics, comorbidities, and insurance status with outcomes. J Gastrointest Surg. 2008;12(2):250-5.
7. Lutfi R, Torquati A, Sekhar N, Richards WO. Predictors of success after laparoscopic gastric bypass: a multivariate analysis of socioeconomic factors. Surg Endosc. 2006;20(6):864-7.

8. Wittgrove AC, Clark GW. Laparoscopic gastric bypass, Roux-enY-500 patients: technique and results, with 3-60 month follow-up. Obes Surg. 2000;10(3):233-9.

9. Higa K, Ho T, Tercero F, Yunus T, Boone KB. Laparoscopic Rouxen-Y gastric bypass: 10-year follow-up. Surg Obes Relat Dis. 2011;7(4):516-25.

10. Magro DO, Geloneze B, Delfini R, Pareja BC, Callejas F, Pareja JC. Long-term weight regain after gastric bypass: a 5 -year prospective study. Obes Surg. 2008;18(6):648-51.

11. Odom J, Zalesin KC, Washington TL, Miller WW, Hakmeh B, Zaremba DL, et al. Behavioral predictors of weight regain after bariatric surgery. Obes Surg. 2010;20(3):349-56.

12. Sarwer DB, Wadden TA, Moore RH, Baker AW, Gibbons LM, Raper SE, et al. Preoperative eating behavior, postoperative dietary adherence, and weight loss after gastric bypass surgery. Surg Obes Relat Dis. 2008;4(5):640-6.

13. Fox B, Chen E, Suzo A, Jolles S, Greenberg JA, Campos GM, et al. Dietary and psych predictors of weight loss after gastric bypass. J Surg Res. 2015.

14. Foster MT, Warne JP, Ginsberg AB, Horneman HF, Pecoraro NC, Akana SF, et al. Palatable foods, stress, and energy stores sculpt corticotropin-releasing factor, adrenocorticotropin, and corticosterone concentrations after restraint. Endocrinology. 2009;150(5): 2325-33.

15. Petereit R, Jonaitis L, Kupcinskas L, Maleckas A. Gastrointestinal symptoms and eating behavior among morbidly obese patients undergoing Roux-en-Y gastric bypass. Medicina (Kaunas). 2014;50(2):118-23.

16. Laurenius A, Larsson I, Bueter M, Melanson KJ, Bosaeus I, Forslund $\mathrm{HB}$, et al. Changes in eating behaviour and meal pattern following Roux-en-Y gastric bypass. Int $\mathrm{J}$ Obes. 2012;36(3):348-55.

17. Miras AD, Al-Najim W, Jackson SN, McGirr J, Cotter L, Tharakan $\mathrm{G}$, et al. Psychological characteristics, eating behavior, and quality of life assessment of obese patients undergoing weight loss interventions. Scand J Surg. 2015;104(1):10-7.

18. Sanchez-Santos R, Del Barrio MJ, Gonzalez C, Madico C, Terrado I, Gordillo ML, et al. Long-term health-related quality of life following gastric bypass: influence of depression. Obes Surg. 2006;16(5):580-5.

19. Karlsson J, Sjöström L, Sullivan M. Swedish obese subjects (SOS) - an intervention study of obesity. Two-year follow-up of health-related quality of life (HRQL) and eating behavior after gastric surgery for severe obesity. Int J Obes Relat Metab Disord. 1998;22(2):113-26.

20. Andersen LF, Tomten H, Haggarty P, Lovo A, Hustvedt BE. Validation of energy intake estimated from a food frequency questionnaire: a doubly labelled water study. Eur J Clin Nutr. 2003;57(2):279-84.

21. Stunkard AJ, Messick S. The three-factor eating questionnaire to measure dietary restraint, disinhibition and hunger. J Psychosom Res. 1985;29(1):71-83.

22. Van Strien T, Frijters JER, Bergers GPA, Defares PB. The Dutch Eating Behavior Questionnaire (DEBQ) for assessment of restrained, emotional, and external eating behavior. Int J Eat Disord. 1986;5(2):295-315.

23. Craig CL, Marshall AL, Sjostrom M, Bauman AE, Booth ML, Ainsworth BE, et al. International physical activity questionnaire: 12 -country reliability and validity. Med Sci Sports Exerc. 2003;35(8):1381-95.

24. Gradaschi R, Camerini G, Carlini F, Sukkar S, Sopinaro N, Adami GF. Physical activity after surgically obtained weight loss: study 
with a Sense Wear armband in subjects undergoing biliopancreatic diversion. Obes Surg. 2013.

25. Unick JL, Bond DS, Jakicic JM, Vithiananthan S, Ryder BA, Roye GD, et al. Comparison of two objective monitors for assessing physical activity and sedentary behaviors in bariatric surgery patients. Obes Surg. 2012;22(3):347-52.

26. Bond DS, Unick JL, Jakicic JM, Vithiananthan S, Pohl D, Roye GD, et al. Objective assessment of time spent being sedentary in bariatric surgery candidates. Obes Surg. 2011;21(6):811-4.

27. Garber CE, Blissmer B, Deschenes MR, Franklin BA, Lamonte MJ, Lee IM, et al. American College of Sports Medicine position stand. Quantity and quality of exercise for developing and maintaining cardiorespiratory, musculoskeletal, and neuromotor fitness in apparently healthy adults: guidance for prescribing exercise. Med Sci Sports Exerc. 2011;43(7):1334-59.

28. Fairburn CG, Beglin SJ. Assessment of eating disorders: interview or self-report questionnaire? Int J Eat Disord. 1994;16(4):363-70.

29. Reas DL, Overas M, Ro O. Norms for the Eating Disorder Examination Questionnaire (EDE-Q) among high school and university men. Eat Disord. 2012;20(5):437-43.

30. Beck AT, Steer RA, Ball R, Ranieri W. Comparison of Beck Depression Inventories-IA and -II in psychiatric outpatients. J Pers Assess. 1996;67(3):588-97.

31. Kolotkin RL, Crosby RD, Kosloski KD, Williams GR. Development of a brief measure to assess quality of life in obesity. Obes Res. 2001;9(2):102-11.

32. Benjamini Y, Hochberg Y. Controlling the false discovery rate: a practical and powerful approach to multiple testing. J R Stat Soc. 1995; Series B(57):289-300.

33. Freire RH, Borges MC, Alvarez-Leite JI, Toulson Davisson Correia MI. Food quality, physical activity, and nutritional follow-up as determinant of weight regain after Roux-en-Y gastric bypass. Nutrition. 2012;28(1):53-8.

34. Faria SL, de Oliveira KE, Lins RD, Faria OP. Nutritional management of weight regain after bariatric surgery. Obes Surg. 2010;20(2):135-9.

35. Welch G, Wesolowski C, Piepul B, Kuhn J, Romanelli J, Garb J. Physical activity predicts weight loss following gastric bypass surgery: findings from a support group survey. Obes Surg. 2008;18(5): 517-24.

36. Josbeno DA, Kalarchian M, Sparto PJ, Otto AD, Jakicic JM. Physical activity and physical function in individuals postbariatric surgery. Obes Surg. 2011;21(8):1243-9.

37. Mundi MS, Lorentz PA, Swain J, Grothe K, Collazo-Clavell M. Moderate physical activity as predictor of weight loss after bariatric surgery. Obes Surg. 2013;23(10):1645-9.

38. Donnelly JE, Blair SN, Jakicic JM, Manore MM, Rankin JW, Smith BK. American College of Sports Medicine position stand. Appropriate physical activity intervention strategies for weight loss and prevention of weight regain for adults. Med Sci Sports Exerc. 2009;41(2):459-71.

39. Warde-Kamar J, Rogers M, Flancbaum L, Laferrere B. Calorie intake and meal patterns up to 4 years after Roux-en-Y gastric bypass surgery. Obes Surg. 2004;14(8):1070-9.
40. Moize V, Andreu A, Flores L, Torres F, Ibarzabal A, Delgado S, et al. Long-term dietary intake and nutritional deficiencies following sleeve gastrectomy or Roux-en-Y gastric bypass in a Mediterranean population. J Acad Nutr Diet. 2013;113(3):400-10.

41. Laurenius A, Larsson I, Melanson KJ, Lindroos AK, Lonroth H, Bosaeus I, et al. Decreased energy density and changes in food selection following Roux-en-Y gastric bypass. Eur J Clin Nutr. 2013;67(2):168-73.

42. Kruseman M, Leimgruber A, Zumbach F, Golay A. Dietary, weight, and psychological changes among patients with obesity, 8 years after gastric bypass. J Am Diet Assoc. 2010;110(4):527-34.

43. Mathus-Vliegen EMH. Long-term health and psychosocial outcomes from surgically induced weight loss: results obtained in patients not attending protocolled follow-up visits. Int J Obes. 2006;31(2):299-307.

44. Burgmer R, Grigutsch K, Zipfel S, Wolf A, de Zwaan M, Husemann B, et al. The influence of eating behavior and eating pathology on weight loss after gastric restriction operations. Obes Surg. 2005;15(5):684-91.

45. Hrabosky J, Masheb R, White M, Rothschild B, Burke-Martindale C, Grilo C. A prospective study of body dissatisfaction and concerns in extremely obese gastric bypass patients: 6- and 12-month postoperative outcomes. Obes Surg. 2006;16(12):1615-21.

46. Castellini G, Godini L, Amedei SG, Faravelli C, Lucchese M, Ricca V. Psychological effects and outcome predictors of three bariatric surgery interventions: a 1-year follow-up study. Eating and weight disorders: EWD. 2014;19(2):217-24.

47. Matini D, Ghanbari Jolfaei A, Pazouki A, Pishgahroudsari M, Ehtesham M. The comparison of severity and prevalence of major depressive disorder, general anxiety disorder and eating disorders before and after bariatric surgery. Med J Islam Repub Iran. 2014;28:109.

48. Strain GW, Kolotkin RL, Dakin GF, Gagner M, Inabnet WB, Christos P, et al. The effects of weight loss after bariatric surgery on health-related quality of life and depression. Nutrition \& diabetes. 2014;4:e132.

49. Kalarchian MA, King WC, Devlin MJ, Marcus MD, Garcia L, Chen JY, et al. Psychiatric disorders and weight change in a prospective study of bariatric surgery patients: a 3-year follow-up. Psychosom Med. 2015.

50. Mitchell JE, King WC, Chen JY, Devlin MJ, Flum D, Garcia L, et al. Course of depressive symptoms and treatment in the longitudinal assessment of bariatric surgery (LABS-2) study. Obesity (Silver Spring, Md). 2014;22(8):1799-806.

51. Faulconbridge LF, Wadden TA, Thomas JG, Jones-Corneille LR, Sarwer DB, Fabricatore AN. Changes in depression and quality of life in obese individuals with binge eating disorder: bariatric surgery versus lifestyle modification. Surg Obes Relat Dis. 2013;9(5):790-6.

52. Averbukh Y, Heshka S, El-Shoreya H, Flancbaum L, Geliebter A, Kamel S, et al. Depression score predicts weight loss following Roux-en-Y gastric bypass. Obes Surg. 2003;13(6):833-6.

53. Nadalini L, Zenti MG, Masotto L, Indelicato L, Fainelli G, Bonora $\mathrm{F}$, et al. Improved quality of life after bariatric surgery in morbidly obese patients. Interdisciplinary group of bariatric surgery of Verona (G.I.C.O.V.). G Chir. 2014;35(7-8):161-4. 